\title{
The LOUVRE Laboratory: State-of-the-Art Ultrafast Ultraviolet Spectroscopies for Molecular and Materials Science
}

\author{
Malte Oppermann, Natalia S. Nagornova, Aurelio Oriana, Edoardo Baldini, Lars Mewes, Benjamin \\ Bauer, Tania Palmieri, Thomas Rossi, Frank van Mourik, and Majed Chergui*
}

\begin{abstract}
We describe the facilities for ultraviolet studies in the femtosecond to nanosecond time domain. These facilities consist of: i) a set-up for deep-ultraviolet spectroscopy in the 260-380 nm range in both pump and probe pulses for transient absorption/reflectivity or two-dimensional spectroscopy studies; ii) a set-up for ultrafast fluorescence measurements with detection down to $300 \mathrm{~nm}$. The capabilities of these set-ups are demonstrated by examples on molecular systems, biosystems, nanoparticles and solid materials.
\end{abstract}

Keywords: Absorption · Charge carriers dynamics · Charge transfer · Deep-UV · Energy relaxation · Fluorescence · Materials · Molecules · Proteins · Reflectivity · Solvation dynamics · Two-dimensional spectroscopy $\cdot$ Ultrafast $\cdot$ Ultraviolet

\section{Introduction}

Driven by the need to describe nonequilibrium phenomena, steady-state spectroscopic tools for the investigation of equilibrium structures continue to be translated into their time-domain analogues in the femtosecond-picosecond regime. During the past three decades, the growing toolbox of ultrafast spectroscopies has successfully established the fields of femtochemistry and femto-biology, ${ }^{[1-6]}$ with a remarkable ability to unravel in real time the structural and relaxation dynamics of electronic and vibrational excited states of molecules, proteins and materials. Pulsed coherent sources from X-rays to terahertz radiation have allowed researchers to investigate ultrafast dynamics via the associated light-matter interactions and gain access to electronic, spin and structural changes from the site-specific atomic to the supra-molecular scale. ${ }^{[7]}$

At the LSU within the Lausanne Centre for Ultrafast Science (LACUS), we have concentrated part of our research efforts on the 250-400 nm spectral range, i.e. the ultraviolet (UV) to deep-UV region. There are several reasons for targeting this range:

${ }^{*}$ Correspondence: Prof. Dr. M. Chergui Lab. de Spectroscopie Ultrarapide (LSU) and Lausanne Centre for Ultrafast Science (LACUS) Ecole Polytechnique Fédérale de Lausanne ISIC, FSB, Station 6

$\mathrm{CH}-1015$ Lausanne

E-mail: Majed.chergui@epfl.ch i) the absorption bands of amino-acid residues such as tryptophan (Trp), tyrosine (Tyr) and phenylalanine (Phe), and of nucleobases such as guanine, cytosine, adenine, etc. lie in this region; ii) the latter is also where several small molecules, organic or inorganic, absorb strongly; iii) it corresponds to the bandgap of semiconductors transition metal oxides (TMO). The latter has attracted much interest in the past twenty years due to their applications in solar energy conversion and in catalysis. While these systems have been investigated in the $\mathrm{THz},{ }^{[8,9]}$ infrared ${ }^{[10]}$ and visible $^{[11-14]}$ regions there have never been studies with deep-UV continuum probes, which can cover the region both above and below the band gap.

The development of ultrafast UV spectroscopic tools has resulted in the LOUVRE (Lots $\underline{\text { Of }} \underline{\mathrm{UV}} \underline{\text { Radiation for your }}$ Experiments) laboratory: a unique university-based facility that comprises various techniques for investigating a wide range of liquid and solid samples. In this article, we present this toolbox of methods and discuss its capabilities by highlighting some of our most recent results. We hope to illustrate the rich contributions of ultrafast UV spectroscopy to a wide range of research fields such as biology, coordination chemistry and materials science.

\section{Experimental Techniques and Set-ups}

The LOUVRE consists of two main set-ups: (1) an ultra-stable, high repetition rate femtosecond laser source for tuneable deep-UV pump and broadband UV con- tinuum probe pulses that are employed in transient absorption (TA) measurements (Fig. 1) or two-dimensional (2D) spectroscopy, and; (2) a high repetition rate, high time-resolution fluorescence up-conversion set-up for ultrafast emission measurements (Fig. 2). Each of these set-ups has its characteristic advantages and offers highly flexible experimental target stations dedicated to different sample types. Most importantly, these two approaches are fully complementary.

In most ultrafast spectroscopic measurements, a first (pump) pulse triggers a photophysical or photochemical process by excitation of the sample, while a second (probe) pulse, whose time delay is tuneable with respect to the pump pulse, using optical delay lines, is used to interrogate the system. In broadband transient absorption (TA), the probe pulse monitors the absorption change of the sample induced by the pump. In the simplest case, the signal (Fig. 1) consists of an induced transparency or ground state bleach (GSB), due to the fact that the ground state has been depopulated, an excited state absorption (ESA) or a stimulated emission (SE). Since the latter and the GSB imply more photons reaching the detector, they have equal signs, while the ESA has an opposite sign (Fig. 1c).

In fluorescence up-conversion (Fig. $2 \mathrm{a}$ ), the second (gate) pulse does not interfere with the sample but it opens a gate at a given time delay in a nonlinear medium which permits the detection of the spontaneous fluorescence, by mixing the gate pulse with the fluorescence and detecting the sum (up-) or difference (down-) frequency of the two. 
(a)

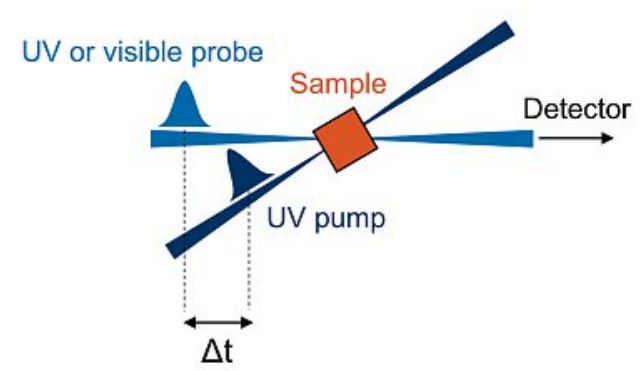

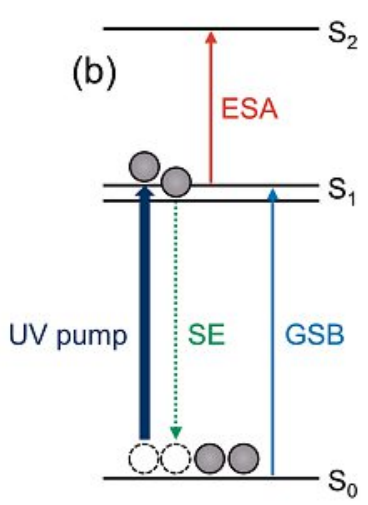

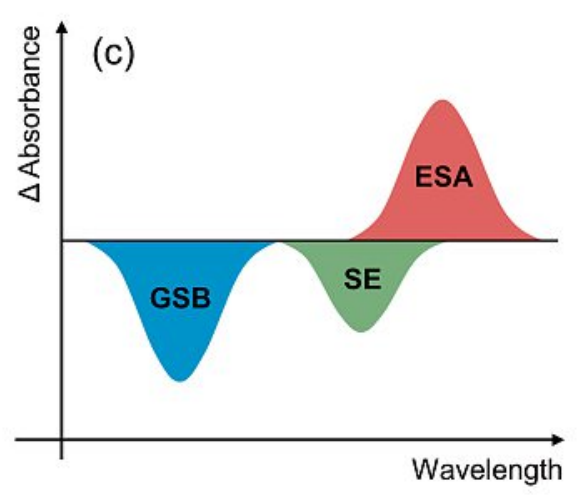

Fig. 1. Schematic illustration of a transient absorption (TA) experiment. (a) typical pump-probe arrangement, where a UV-pump pulse interacts with a sample and the transmitted intensity of a broadband Visible- or UV-probe pulse is measured using a detector (a photomultiplier, PMT, for example). (b) processes contributing to the idealised TA spectrum displayed in (c). When a UV-pump transfers population from a ground state ( $\mathrm{S}_{0}$ ) to an excited state $\left(S_{1}\right)$, the absorption corresponding to the $S_{0}-S_{1}$ transition is reduced. This leads to a negative ground state bleach (GSB) feature in the transient absorption spectrum. Analogously, excited state absorption (ESA) and stimulated emission (SE) may take place from the transiently populated state $\mathrm{S}_{1}$.

(a)

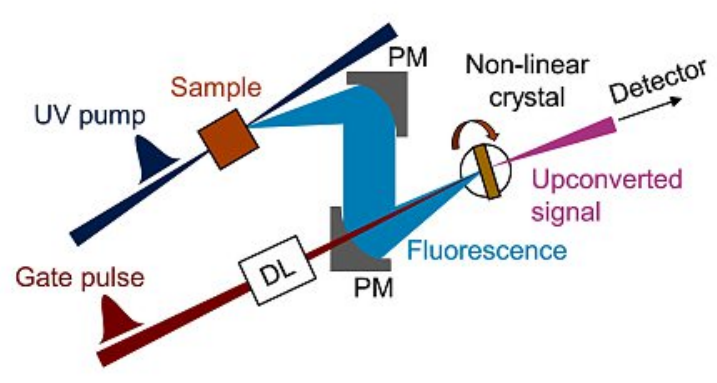

(b)

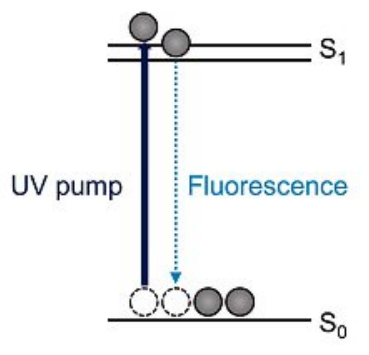

(c)

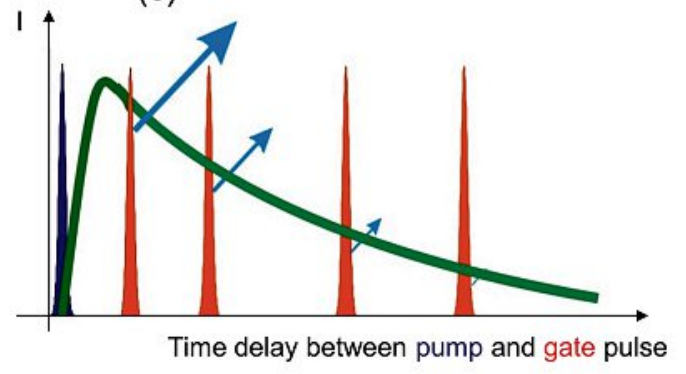

Fig. 2. Schematic illustration of a fluorescence up-conversion (FIUC) experiment. (a) A simplified experimental set-up, where a UV-pump induces fluorescence in a sample. The corresponding energy level scheme is displayed in (b). The emission is collected and focused into a nonlinear medium, where it interacts with a gating pulse. The up-converted signal is then detected by a photomultiplier (PMT) or a CCD camera. (c) Principle of a fluorescence time-gating: at $t=0$ the pump pulse (purple) excites the sample and triggers a spontaneous fluorescence of the sample with a given time profile (green trace). This fluorescence is mixed within the gate pulse (orange) whose time delay with respect to the pump is tuneable. At each time delay the sum frequency of the fluorescence with the gate pulse yields the up-converted light (blue arrows) whose time profile reflects that of the fluorescence.

\subsection{Two-dimensional UV Spectroscopy}

In the past twenty years, there has been a drive aimed at extending the methods of multidimensional Fourier transform NMR spectroscopy into the shorter wavelength range. ${ }^{[4,5,15]}$ This was achieved first in the infrared, ${ }^{[6]}$ then in the visible region. ${ }^{[3]}$ The aim of such developments is to reveal correlations and interactions between the vibrational or electronic dipoles probed as cross-peaks in a two-dimensional map, which typically plots the probe frequency as a function of the excitation frequency. In this way, one may obtain information on electron and/or energy transfer processes and conformational changes encoded in the electronic dipole couplings. This is illustrated in Fig. 3, where the idealized case of two coupled electronic transitions and the associated two-dimensional spectrum is explained. In addition, homogeneous and inhomogeneous contributions can be extracted from the two-dimensional line shapes, such that their temporal evolution can be used to study the interaction between a system and its environment, e.g. a solute inside a solvent.

The interest in 2DUV spectroscopy is obvious for the study of the structure and dynamics of biosystems with a complex molecular architecture.[6,16,17] Extending nonlinear optical spectroscopy techniques into the UV region is a particularly challenging technological task, which originates from the increased requirements regarding phase- and dispersion-control at short wavelengths. Nevertheless, throughout the past decade impressive progress has been made, which is presented in the excellent review by Cannizzo. ${ }^{[18]}$ There are two approaches to this type of spectroscopy: the Fourier transform (FT or coherent) one and the transient absorption (or incoherent) one. In the former, the excitation frequency is resolved by varying the delay between two ultrashort pump pulses and performing the FT of the time-dependent nonlinear signal. ${ }^{[19,20]}$ The main technical challenge of this method is the generation of phase-locked, temporally compressed pulses, required to perform a clean FT of the nonlinear signal without distorting the two-dimensional maps. Despite these challenges, the access to phase information and thus to vibrational and electronic coherences makes it a particularly rewarding approach. ${ }^{[21-25]}$ However, as UV absorption bands of chromophores are often broad, covering their entire profile of typically $50 \mathrm{~nm}$ requires a $\sim 3$ fs pulse at $300 \mathrm{~nm}$, which is quite challenging. The second approach is usually referred to as 2DUV TA, and it uses a single tuneable narrowband pump pulse to sequentially acquire TA spectra with a broadband UV continuum probe. Whilst lacking phase information, this technique neither requires transform limited pulses nor their phase control. Its main advantages are a much simpler and more robust set-up at the cost of a reduced time resolution. However, the latter is not 


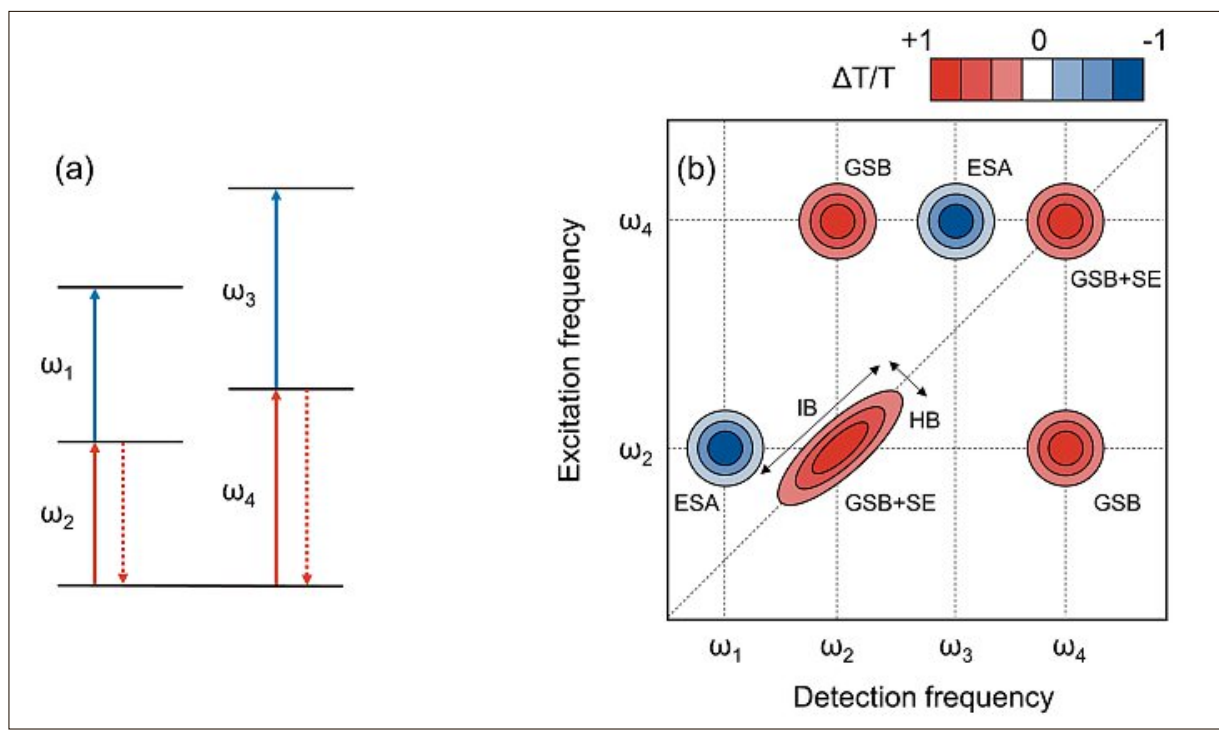

Fig. 3. Principle of multidimensional spectroscopies. (a) Energy level scheme of two coupled oscillators and (b) relative two-dimensional map of the differential transmission signal. In (a) solid red arrows correspond to a ground-state bleach (GSB), dashed red arrows to stimulated emission (SE) and blue arrows to excited state absorption (ESA). In (b) red signals are positive, blue signals are negative. Homogeneous broadening (HB) and inhomogeneous broadening (IB) are highlighted in the peak along the diagonal. Figure reproduced from ref. [62]. a stringent requirement, especially in biological systems where interactions between chromophores can be long-range and therefore, occur over rather long time scales.

The latter approach was implemented at the LOUVRE lab. Despite not being a pump frequency scan, we circumvent long data acquisition times through the use of an ultra-stable, high repetition rate femtosecond UV pulse source. The associated broadband TA set-up (Fig. 4) accommodates probe pulse configurations both in the visible and deep-UV regions and has been described in detail elsewhere. ${ }^{[26,27]}$ It delivers broadband visible femtosecond pump pulses (520-740 nm bandwidth, $15 \mu \mathrm{J}$ per pulse) that can be used as such or be frequency doubled to generate deepUV narrowband $(1.5 \mathrm{~nm})$ pump pulses via second harmonic generation (SHG) in different $\beta$-barium borate (BBO) crystals. For the probe, a so-called achromatic doubling scheme is employed, ${ }^{[28]}$ where broadband phase matching is achieved by spatially chirping the beam before tightly focussing it into a thin BBO crystal. This routinely provides pulses with bandwidths $>100 \mathrm{~nm}$ in the deep-UV. This configuration represents the optimal conditions for pump-probe 2DUV spectroscopy with an instrument response function (IRF) of 150 fs. Nevertheless, improved time resolution down to $50 \mathrm{fs}$ has been achieved by narrowing the visible NOPA output spectrum or by separately compressing the visible pump pulses before SHG. ${ }^{[29]}$ In addition, part of the $800 \mathrm{~nm}$ output can be used as a pre-pulse or pump (insingle-shot technique, due to the required cluding its third and second harmonic) or to generate a white-light continuum probe for visible TA. For the detection, a fibre-coupled imaging spectrograph is used in conjunction with a fast, double-array multichannel detector. This allows shot-to-shot recording of the probe pulse spectrum together with a reference beam at an exceptional data-acquisition rate of
$20 \mathrm{kHz} .{ }^{[27]}$ As a result, absorbance changes $<1 \mathrm{mOD}$ can be resolved after comparatively short measurement times.

The flexibility of this set-up is exemplified by the large diversity of systems and flow cells or wire-guided liquid jet allows the investigation of liquid samples, while a transient reflectivity configuration enables the study of single crystals.

\subsection{Time-resolved Fluorescence}

Femtosecond fluorescence up-conversion (FlUC) spectroscopy is a widely used technique for studying intramolecular electronic relaxation processes, vibrational cooling and solvation dynamics of molecular systems. It is especially used when the fluorescence decay times are less than a few ps, a time scale that cannot be resolved with detectors such as multichannel plates or streak cameras cannot resolve the decay. This approach represents a simplification compared to TA because the sample interacts with the excitation pulse only (Fig. 2). The operating principle of our set-up is shown in Fig. 5. The fluorescence is collected by wide-angle optics and focused onto a nonlinear sum-frequency (SF) crystal in which it is mixed with the gate pulse, whose time delay with respect to the pump pulse is controlled by an optical delay stage. The intensity of the sum-frequency (up-converted) light is then recorded as a function of delay time between pump and gate pulses. With reflective optics to miniprocesses we investigated. Here, the use of

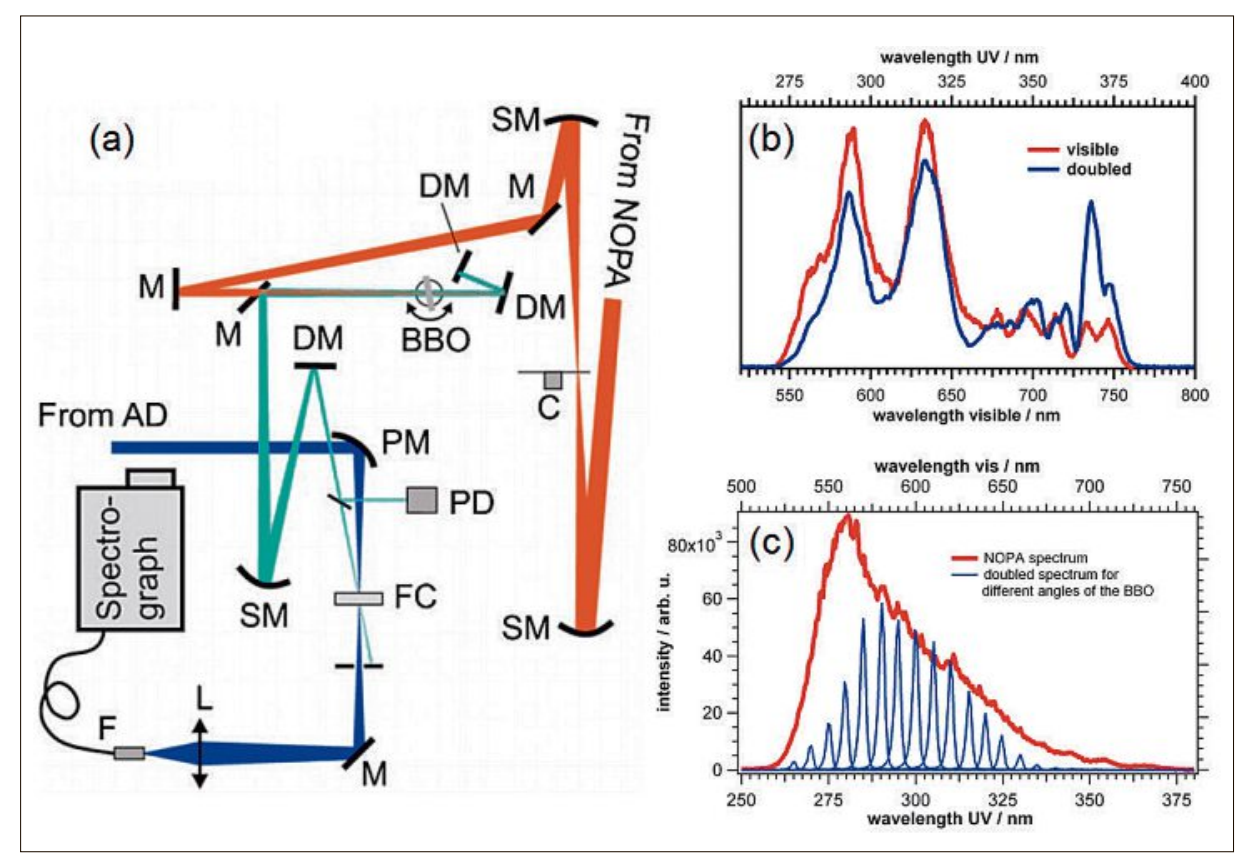

Fig. 4. Optical beamline (a) and performance of the probe pulse generation via achromatic doubling (AD) displayed in (b). The pump pulse generation via tuneable narrowband doubling is shown in (c). The probe pulses (dark blue line) are focussed via a parabolic mirror (PM) into the sample flow cell (FC) and the transmitted intensity is collected via a fibre-coupled (F) spectrograph. The pump pulses from the NOPA (orange line) are optically chopped by a chopper (C) and frequency doubled via a tuneable BBO (light blue line). The remaining visible light is filtered via dichroic mirrors (DM) and focussed on the sample via a spherical mirror (SM). Reproduced from ref. [26]. 
mize frequency dispersion effects after the sample, a time resolution of 100-130 fs can be reached. ${ }^{[30]}$ To accomplish a broadband detection, the up-converted signal, at fixed time delay, is detected with a spectrograph equipped with a charge coupled device (CCD) camera, and the SF crystal is rotated during the integration time at a constant angular speed to cover the spectral range of interest. The peculiarity of our set-up lies in the fact that it extends from the IR (between 1 and $2 \mu \mathrm{m})^{[31]}$ to the $\mathrm{UV}^{[30]}$ (above $300 \mathrm{~nm}$ ) spectral domains.

\section{Research Highlights}

\subsection{Biological Systems}

UV-chromophores are site-specific probes of their local environment. [32] This is especially the case for tryptophan (Trp), whose absorption and emission bands are centred at about $280 \mathrm{~nm}$ and $360 \mathrm{~nm}$, respectively. For example, as part of a donoracceptor pair undergoing Fluorescence Resonance Energy Transfer (FRET), its fluorescence decay is routinely used to determine the geometrical arrangement of the chromophore pair with nm resolution. ${ }^{[33]}$ In addition to its capacity to act as a probe for energy and transfer processes, Trp is also a sensor of local electric fields inside a protein. ${ }^{[34,35]}$ In this spirit, we have applied our UV-spectroscopy tools to probe the energy and charge transfer processes in hemoproteins, such as horse heart cytochrome $\mathrm{c}(\mathrm{Cyt} \mathrm{c})^{[36,37]}$ and myoglobins. [38-40]

In refs [36] and [37] we investigated the interaction between the heme and a single Trp residue in Cyt $\mathrm{c}$ in its ferric and ferrous redox states. A single Trp residue, which is located very close to the heme and known to undergo very efficient energy transfer to it, makes Cyt c an ideal model system to study the photodynamics of this protein class in its two different redox states. Indeed, we showed, using time-resolved fluorescence, that excited Trp decays ${ }^{[36]}$ with a much shorter time constant (350 fs) in the ferrous state than in the ferric case (770 fs). This implies a more efficient energy transfer in the former case, which may be due to the significantly different relative orientations and distances between the Trp and the heme groups in the two redox states, which is supported by computations and (CD) measurements. ${ }^{[41,42]}$ Exciting the heme did not show a response of the Trp contrary to what we had observed in bacteriorhodopsin. ${ }^{[34,35]}$ This result is surprising and it calls for further investigation as the Trp decay times reflect a strong interaction with the heme. In addition, the much shorter Trp decay times in Cyt c are to be contrasted to the case of myoglobins, which we discuss hereafter.

Myoglobin $(\mathrm{Mb})$ belongs to the family of heme proteins and consists of 153 amino acids and an active iron porphyrin (heme) centre. It contains two tryptophan residues: $\operatorname{Trp}^{7}$ and $\operatorname{Trp}^{14}$, which are located in the $\alpha$-helix $A$ with respective distances of $21.2 \AA$ and $15.2 \AA$ (centre to centre) to the heme group. In both the ferrous $\left(\mathrm{Fe}^{2+}\right)$ and the ferric state $\left(\mathrm{Fe}^{3+}\right)$, the metal atom can bind a variety of diatomic ligands (including $\mathrm{O}_{2}, \mathrm{CO}$, $\mathrm{NO}$ and $\mathrm{CN}$ ), which determines its function. Photoexcitation of the Trp residues in Mb leads to its fluorescence, with much shortened decay times compared to the isolated case $(c a .3 \mathrm{~ns})$ :

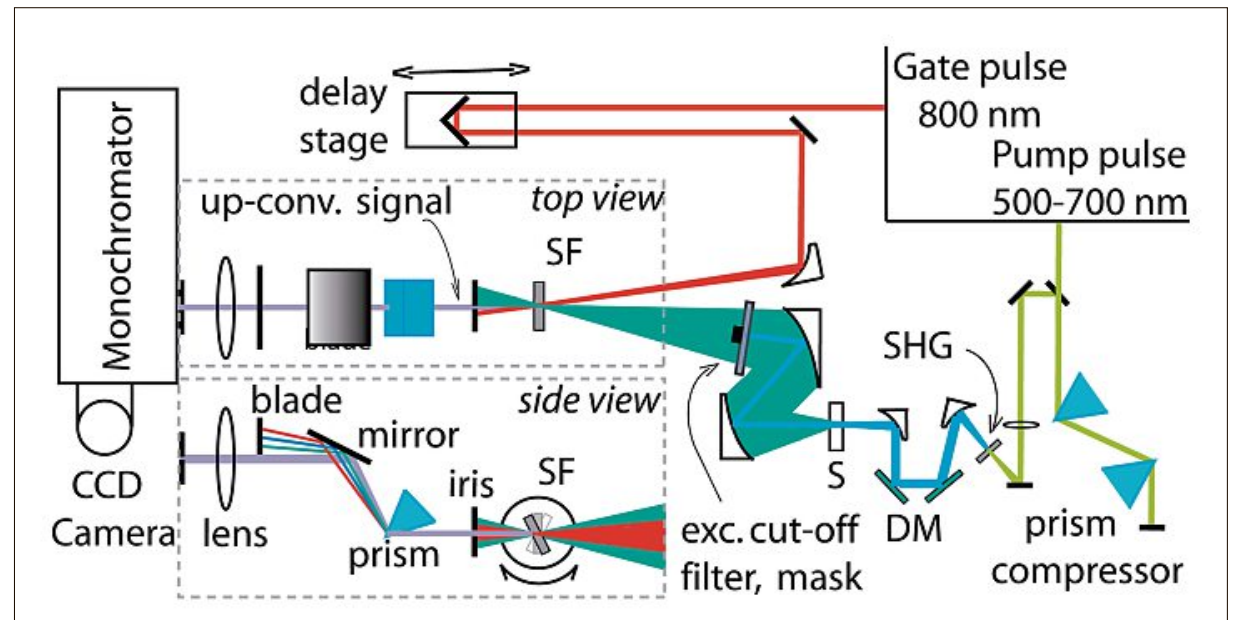

Fig. 5. Schematics of the time-gated fluorescence up-conversion experimental set-up. After excitation of the sample (S), spontaneous emission is collected and focused by two parabolic mirrors onto a sum-frequency (SF) crystal. The broad emission spectrum is then time-gated by the delayed pulses, throughout the continuous rotation of the SF crystal. After spatial filtering of scattered light, the up-converted signal is dispersed by a grating inside of a monochromator and finally detected by a liquid nitrogen cooled, UV-enhanced CCD camera. For the sake of clarity, the SFG process and the signal filtering parts are shown from the top and form the side. Reproduced from ref. [30].

20 to 30 ps for $\operatorname{Trp}^{14}$ and 110 to 140 ps for $\operatorname{Trp}^{7} \cdot{ }^{[43-45]}$ These have been attributed to FRET to the heme. ${ }^{[33,45]}$ Using our 2DUV set-up, we revealed that in ferric myoglobins $\mathrm{MbCN}$ and metMb, ${ }^{[39]} \operatorname{Trp}^{14}$ undergoes a hitherto unsuspected partial electron transfer to the heme, while relaxation of the more distant $\operatorname{Trp}^{7}$ is indeed due to FRET to the heme (Fig. 6). The electron transferred from $\operatorname{Trp}^{14}$ ends up on the iron atom forming a ferrous porphyrin. We then extended these studies to the case of the ferrous (unligated) deoxy form, ${ }^{[40]}$ and found the same $\operatorname{Tr}^{14}$-to-heme electron transfer, this time leading to the formation of a low-valence porphyrin anion radical. More recent (unpublished) work on ligated ferrous Mbs reveals the same pattern of Trp-to-heme electron transfer. In ref. [40], we suggested that the pathway for the electron transfer proceeds via the leucine $69\left(\mathrm{Leu}^{69}\right)$ and valine $68\left(\mathrm{Val}^{68}\right)$ residues, which are in van der Waals contact with each other, while $\mathrm{Leu}^{69}$ is in van der Waals contact with $\operatorname{Trp}^{14}$. In a recent theoretical modelling of the Trp-heme electron transfer Suess et al. confirmed our hypothesis. ${ }^{[46]}$ The above results of a Trp-mediated electron transfer competing with FRET are especially important for the fundamental understanding of electron transfer in biological systems, but also show a limitation of Trp as a spectroscopic ruler in FRET studies of protein dynamics. Indeed, evidence that FRET is taking place requires not only the decay of the donor fluorescence, but also the detection of the acceptor luminescence, a criterion that is often overlooked in studies of protein dynamics.

\subsection{Coordination Chemistry}

Transition metal complexes possess different types of states such as: metal-to-ligand charge transfer (MLCT), metal centred (MC), ligand centred (LC) and ligand-to-metal charge transfer (LMCT). The MLCT state is often the lowest dipole-allowed electronic transition. These complexes are involved in several applications such as solar cells, OLEDs, molecular electronics, biology, magnetic data storage, etc., for which the understanding of the underlying ultrafast photophysics is essential.

Fluorescence up-conversion is ideal in this respect and as an example we show here the case of the cascade of electronic states in an iridium complex, $\operatorname{Ir}(\mathrm{ppy})_{3}$, upon $266 \mathrm{~nm}$ excitation of its high-lying ligand-centred (LC) electronic state. ${ }^{[47]}$ The luminescence of $\operatorname{Ir}(\mathrm{ppy})_{3}$ in DMSO upon $266 \mathrm{~nm}$ excitation of its LC state is shown in Fig. 7 as a function of wavelength and time delay. The LC fluorescence, centred at $330 \mathrm{~nm}$, decays in $70 \pm 10 \mathrm{fs}$, which is significantly shorter than the lifetime of ppy ligands in solution $(\sim 1 \mathrm{~ns}) .{ }^{[48]}$ The LC state 


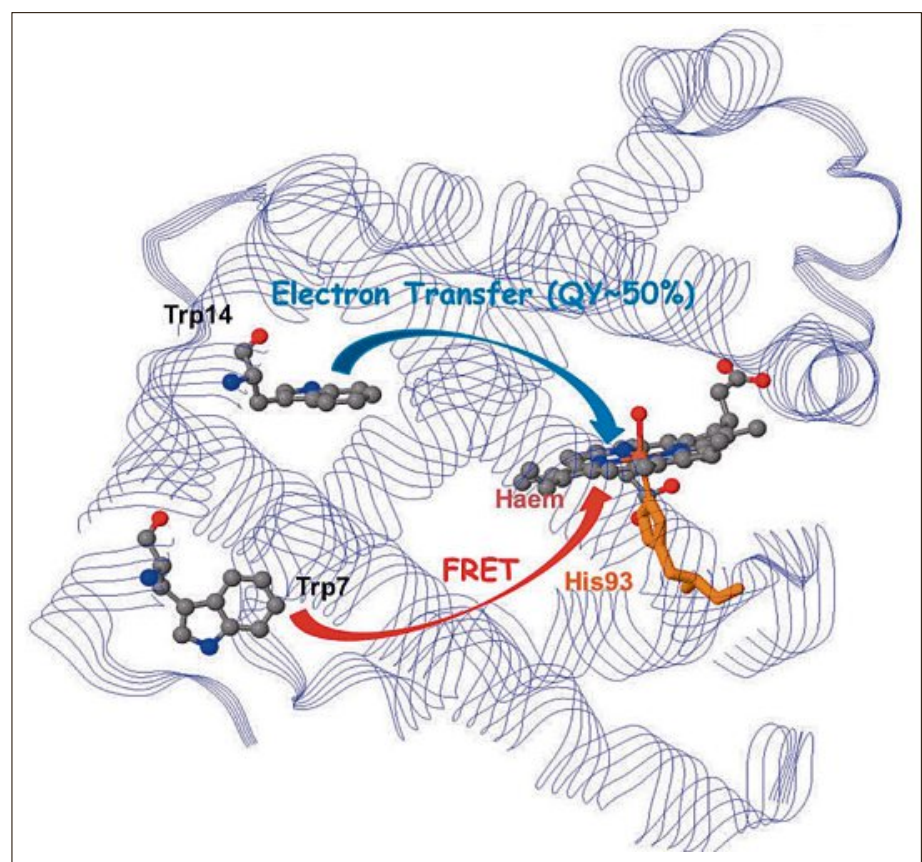

Fig. 6. 2DUV transient absorption of myoglobins revealed that while Trp-7 decays via FRET to the heme, Trp14 undergoes electron transfer with a yield of ca. $50 \%$ while the rest decays by FRET (see refs $[39,40])$.

then decays to the manifold of close-lying spin-mixed MLCT and MC states which undergo an ultrafast ( $<10 \mathrm{fs}$ ) intramolecular electronic-vibrational relaxation, leading to population of the high vibrational levels of the lowest ${ }^{3}$ MLCT state, whose population rises in $70 \mathrm{fs}$. We are therefore able to observe the departure, the intermediate steps, and the arrival of the relaxation cascade that spans more than $1.6 \mathrm{eV}$, from the ${ }^{1} \mathrm{LC}$ state to the lowest ${ }^{3} \mathrm{MLCT}$ state, yielding the long-lived luminescence of the molecule. These results represent the first measurement of the complete relaxation time over an entire cascade of electronic states in a polyatomic molecule. The most remarkable result is that intramolecular electronic relaxation proceeds at sub-vibrational time scales $(<10 \mathrm{fs})$ over a large energy gap. This was already hinted at in our previous studies on organic and inorganic molecular complexes, ${ }^{[49,50]}$ but the occurrence of LC emission in the present case provides a 'clock' of the relaxation dynamics. Such ultrafast electronic 'cooling' does not mean that the molecule is 'cold' in absolute terms. The excess electronic energy is impulsively converted into

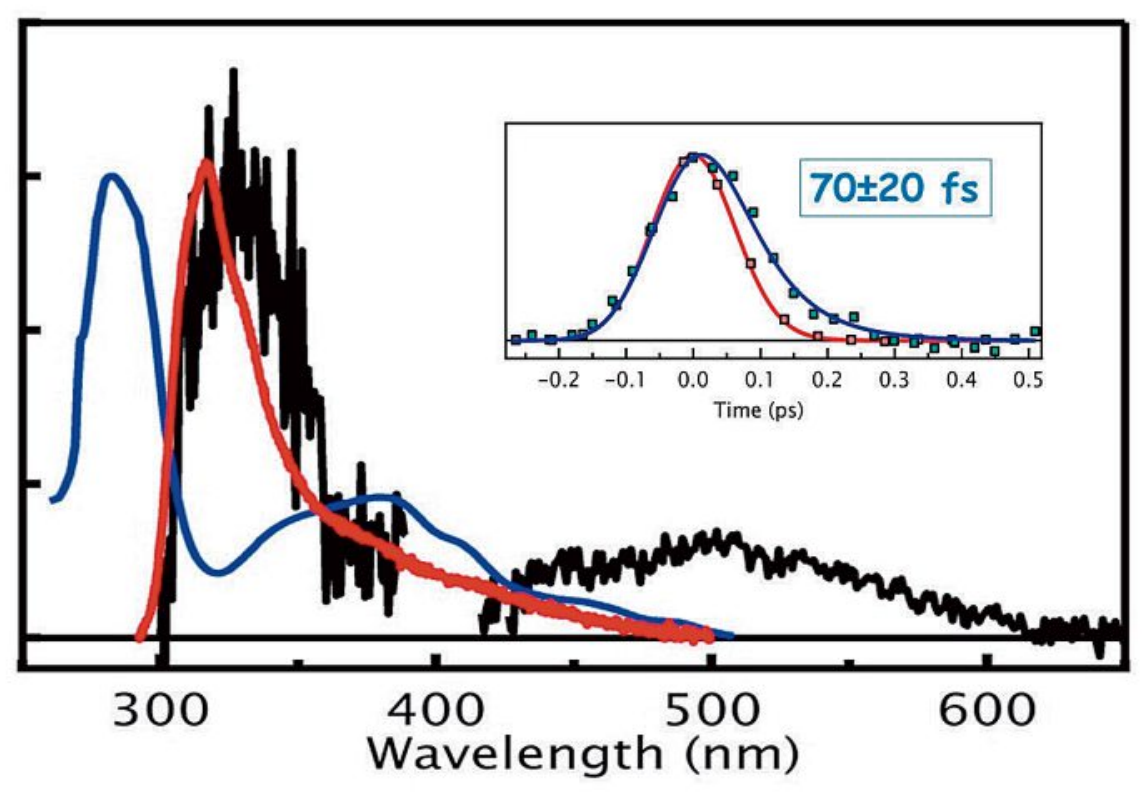

Fig. 7. Absorption spectrum of fac-tris(2-phenylpyridine)-iridium(III) or $\operatorname{Ir}(\mathrm{ppy})_{3}$ in a DMSO solvent and time zero fluorescence spectrum (black) upon excitation of the LC band at $266 \mathrm{~nm}$. The red trace represents the steady-state fluorescence spectrum of the ppy ligand and the blue trace the steady-state absorption spectrum. The inset compares the cross-correlation (red Gaussian), which is the Raman signal of the solvent, with the time profile measured on the emission at 340 $\mathrm{nm}$. Reproduced from ref. [47]. low frequency vibrational modes of the molecule, which are often optically silent ones. The most outstanding outcome of these studies is that electronic 'cooling' of large energy gaps occurs at sub-vibrational time scales. This issue calls for further investigations with methods at even higher time resolution, eventually going into the attosecond regime.

\subsection{Materials Science: Transition Metal Oxides}

2DUV TA spectroscopy offers new perspectives in the field of materials science, shedding light on the elusive physics of strongly interacting and correlated quantum systems. Using this non-equilibrium spectroscopic approach, one can aim at: 1) discovering new hidden or metastable high-energy excitations in a given material; 2) revealing how the low-energy excitations affect specific high-energy collective modes (e.g. excitons...) of the system. In this respect, ultrafast broadband UV spectroscopy becomes a superior tool for unravelling complex phenomena in TMOs, a class of materials in which a plethora of effects emerges due to the non-trivial interplay between low- and high-energy degrees of freedom.

Anatase $\mathrm{TiO}_{2}$ is a prototypical TMO used in a number of applications (e.g. photocatalysis, photovoltaics, etc.). Recently, the application of equilibrium spectroscopies and many-body perturbation theory calculations revealed that the absorption threshold of this material is dominated by strongly bound direct excitons rising over the continuum of indirect interband transitions. ${ }^{[51]}$ These excitons are confined on a two-dimensional (2D) plane of the three-dimensional crystal lattice and remain stable at room temperature in the case of single crystals. However, in all applications, highly defective samples (e.g. nanoparticles, NPs) are used at room temperature and ambient pressure. One may therefore question the existence of these excitons for the actual systems used in technology. Indeed, the equilibrium absorption spectrum of colloidal anatase $\mathrm{TiO}_{2}$ NPs does not show obvious signatures of excitonic transitions, and is rather featureless (see black dotted trace in Fig. 8a). A powerful approach to address the existence of excitonic transitions in a material is to interrogate the system out-of-equilibrium via ultrafast broadband optical spectroscopy. Typically, the exciton line shapes can be identified through the pump-induced transparency of the excitonic peak, referred to as 'exciton bleaching'. To clearly observe this effect in colloidal solutions of anatase $\mathrm{TiO}_{2}$ NPs, ultrafast TA UV spectroscopy is a superior technique because it subtracts the scattered light and provides a better contrast for resolving 
possibly hidden features. By applying this technique on single crystals (in reflectivity mode) and nanoparticles (in transmission mode), the excitonic species could be clearly revealed (Fig. 8). ${ }^{[51]}$ Fig. 8b shows the results of transient reflectivity measurements of single crystals after converting them to absorption changes. The data is dependent on the crystal orientation, showing that the first peak is dominant along the a-axis, while the second band is dominant along the c-axis. More details are found in ref. [51]. These results show that the excitons are very robust against defects, an aspect that has strong consequences in the field of applied research, since the excitons can store the incoming energy in the form of light and guide it at the nanoscale in a selective way.

These measurements have helped clarify the single-particle and many-body effects leading to the exciton bleach and to track the ultrafast intra-band electron dynamics. ${ }^{[29]}$ So far, they had also been elusive to experimental probes and become accessible only when the broadband probe is tuned to cover a broad UV spectral range between 280 and $360 \mathrm{~nm}$.

These first results demonstrate the power and versatility of ultrafast 2DUV spectroscopy for the investigation of a vari- ety of quantum phenomena in complex oxides. Extensions of this technique involve the study of direct excitons in $\mathrm{ZnO},{ }^{[52]}$ the mapping of the charge dynamics across the charge-transfer gap in $\mathrm{NiO},{ }^{[53]}$ and the unravelling of spin polarons in $\mathrm{LaSrMnO}_{4}{ }^{.54]}$

\subsection{Solvation Dynamics}

The first step of an intermolecular charge transfer (CT) reaction (e.g. electron or proton transfer) between a donor and an acceptor in solution is a charge transfer to the solvent. Charge-transfer-to-solvent (CTTS) states, which are quasi-bound states of the solute-solvent system with no equivalent for the isolated ions, are ideal objects to investigate this first step. CTTS states are common in the case of aqueous halides and, since the latter lack internal (nuclear) degrees of freedom, the CTTS dynamics is entirely dependent on the structure and motion of the solvent species. CTTS states are therefore ideal probes of electronic solvation dynamics upon electronic excitation of a solute.

Ultrafast TA and photoemission (PE) spectroscopies have been employed to observe the early-time dynamics of aqueous CTTS states. ${ }^{[55-59]}$ It has been shown that in water, the electron is detached from the iodide in $\sim 0.2$ ps followed by an approx.

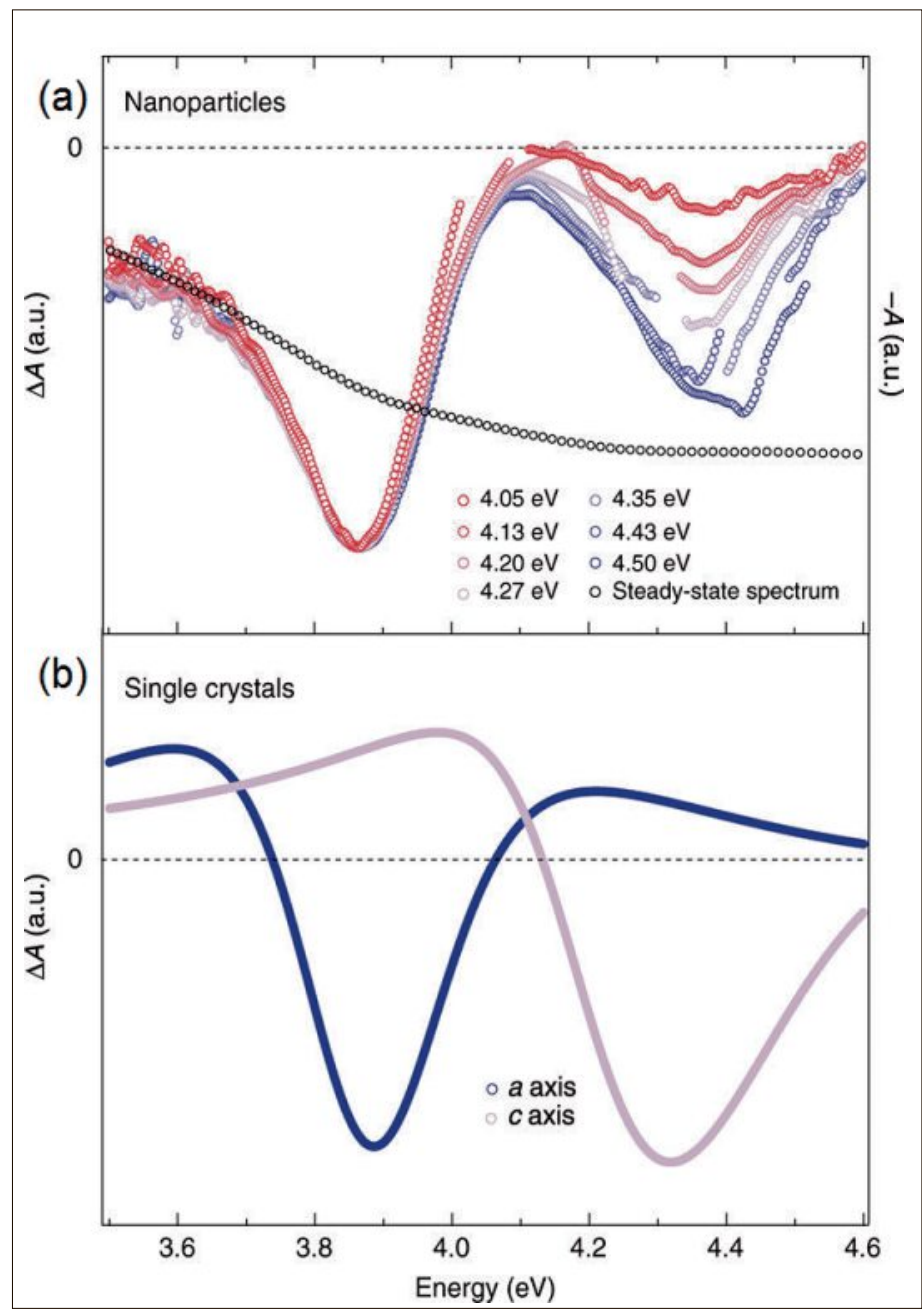

Fig. 8. (a) Normalized transient absorption $(\Delta \mathrm{A})$ spectra at room temperature of a colloidal solution of anatase $\mathrm{TiO}_{2} \mathrm{NPS}$ at a fixed time-delay of 1 ps and for different pump photon energies (indicated in the figure). Each trace is normalized with respect to the minimum of the main feature at $3.88 \mathrm{eV}$. For comparison, the black trace shows the inverted steady-state absorption spectrum. (b) $\Delta$ A spectra (converted from transient reflectivity measurements) of room temperature anatase $\mathrm{TiO}_{2}$ single crystals along the a- and c-axis at a fixed time delay of 1 ps. For this experiment, the pump photon energy is 4.40 $\mathrm{eV}$. Reproduced from ref. [51].
1 ps relaxation of the solvent cage around the aqueous electron ${ }^{[58,59]}$ but the strong absorption (in TA) or PE signal of the solvated electron have hindered the observation of concurrent dynamics of the solute. Fluorescence of the CTTS states would provide a direct measurement of the electron departure to the solvent since photons are emitted during the time the ground and the excited (CTTS) state wave functions of the solute overlap. We reported for the first time the observation of CTTS fluorescence using deep-UV FIUC in the case of aqueous $\mathrm{I}^{-} \cdot{ }^{[60]}$

The fluorescence spectrum of aqueous iodide, measured upon excitation at 266 $\mathrm{nm}$, is shown in Fig. 9. An emission spanning from the UV (approx. $300 \mathrm{~nm}$ ) to the visible region (approx. $670 \mathrm{~nm}$ ) appears promptly at time zero. Its decay is strongly wavelength-dependent going from $\mathrm{ca}$. 60 fs at $\lambda<330 \mathrm{~nm}$ to $\sim 50 \mathrm{fs}$ at $650 \mathrm{~nm}$. These results reveal the very large inhomogeneity of the excited centres with each iodide having a somewhat different solvent shell at $\mathrm{t}=0$. The starting solvent configuration will determine the subsequent dynamics where the solvent accommodates to the excited species giving rise to very different Stokes shifts spanning over $1 \mathrm{eV}$. The redder the emission, the larger the Stokes shift and, therefore, the more stable the configuration, which is reflected in a longer decay time. The various time scales, in particular in the redder part of the spectrum could not be observed by TA or other methods because the signal of the solvated electron overshadows these dynamics.

\section{Conclusions}

The present contribution reported on the applications of ultrafast deep UV spectroscopies, either in transient absorption, reflectivity, and in fluorescence. The availability of high performance set-ups allows the study of a wide range of systems, for which prominent deep-UV bands are present. The examples of biological systems and transition metal oxides presented here underscore the importance of covering this spectral range. In the case of transition metal oxides, the ability to excite either the first excitons or higher into the conduction band allows for a distinction between the dynamics of excitons as opposed to that of free carriers. Concerning molecular systems, several studies are now possible for which the photoinduced processes are excitation wavelength dependent, e.g. the recently investigated case of aqueous $\left[\mathrm{Fe}(\mathrm{CN})_{6}\right]^{4-} .{ }^{[61]}$ Developments are undergoing aimed at time-resolved deep-UV circular dichroism, which can be applied to studies of chirality or conformational changes in proteins. Finally, pushing 


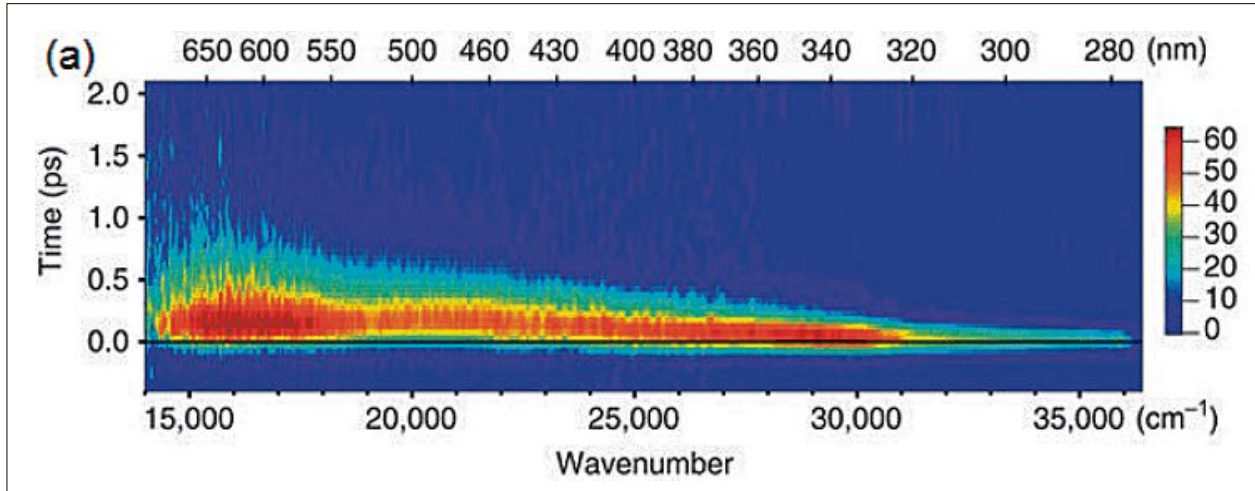

(b)

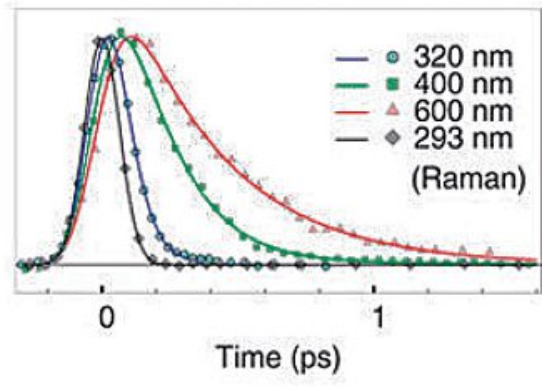

Fig. 9. Femtosecond fluorescence of aqueous iodine. (a) Fluorescence of $1 \mathrm{M} \mathrm{Nal}$ dissolved in water upon $266 \mathrm{~nm}$ excitation. The Raman signal from water was removed from the plot. (b) Normalized kinetic traces at different wavelengths with their representative fits (continuous lines), compared with the Raman signal from water at $293 \mathrm{~nm}$, whose temporal width gives the IRF of the set-up. Reproduced from ref. [60].

further into the shorter wavelength range opens the door to the exploitation of the strong peptide absorptions in proteins.

\section{Acknowledgements}

We acknowledge financial support from the Swiss NSF via the NCCR:MUST and contracts No. 206021_157773 and 20020_153660 as well as the European Research Council Advanced Grants H2020 ERCEA 695197 DYNAMOX.

Received: April 18, 2017

[1] A. H. Zewail, J. Phys. Chem. A 2000, 104 5660.

[2] S. K. Pal, A. H. Zewail, Chem. Rev. 2004, 104 2099

[3] Y. C. Cheng, G. R. Fleming, Annu. Rev. Phys. Chem. 2009, 60, 241

[4] M. D. Fayer, Annu. Rev. Phys. Chem. 2009, 60 , 21

[5] R. J. D. Miller, A. Paarmann, V. I. Prokhorenko, Acc. Chem. Res. 2009, 42, 1442.

[6] P. Hamm, M. T. Zanni, 'Concepts and Methods of 2D Infrared Spectroscopy', Cambridge University Press, 2011.

[7] M. Chergui, 'Comprehensive Biophysics, Vol. 1, Biophysical Techniques for Structura Characterization of Macromolecules', Eds E. H. Egelman, H. J. Dyson, Oxford, Academic Press, 2012, pp. 398-424.

[8] E. Hendry, M. Koeberg, J. Pijpers, M. Bonn, Phys. Rev. B 2007, 75, 233202.

[9] H.-K. Nienhuys, V. Sundström, Appl. Phys. Lett. 2005, 87, 012101

[10] J. B. Asbury, E. Hao, Y. Wang, H. N. Ghosh, T. Lian, J. Phys. Chem. B 2001, 105, 4545.

[11] Y. Tamaki, K. Hara, R. Katoh, M. Tachiya, A Furube, J. Phys. Chem. C 2009, 113, 11741.

[12] M. Rasmusson, A. N. Tarnovsky, T. Pascher, V. Sundström, E. Åkesson, J. Phys. Chem. A 2002 106,7090 .

[13] A. N. Tarnovsky, M. Wall, M. Gustafsson, N Lascoux, V. Sundström, E. Åkesson, J. Phys. Chem. A 2002, 106, 5999.

[14] G. Benko, J. Kallioinen, J. E. Korppi-Tommola A. P. Yartsev, V. Sundström, J. Am. Chem. Soc 2002, 124, 489

[15] R. R. Ernst, G. Bodenhausen, A. Wokaun, 'Principles of Nuclear Magnetic Resonance in One and Two Dimensions', Clarendon Press, Oxford University Press, Oxford, New York, 1987.

[16] D. M. Jonas, Science 2003, 300, 1515.
[17] M. Cho, 'Two-Dimensional Optical Spectroscopy', CRC Press, 2009.

18] A. Cannizzo, Phys. Chem. Chem. Phys. 2012 14, 11205.

[19] W. P. de Boeij, M. S. Pshenichnikov, D. A Wiersma, Chem. Phys. Lett. 1995, 238, 1

[20] L. P. DeFlores, R. A. Nicodemus, A. Tokmakoff, Opt. Lett. 2007, 32, 2966.

[21] B. A. West, A. M. Moran, J. Phys. Chem. Lett. 2012, 3, 2575 .

[22] C. Tseng, S. Matsika, T. C. Weinacht, Opt Express 2009, 17, 18788.

[23] U. Selig, C.-F. Schleussner, M. Foerster, F Langhojer, P. Nuernberger, T. Brixner, Opt Lett. 2010, 35, 4178 .

[24] N. Krebs, I. Pugliesi, J. Hauer, E. Riedle, New J. Phys. 2013, 15, 085016

25] R. Borrego Varillas, A. Oriana, L. Ganzer, A. Trifonov, I. Buchvarov, C. Manzoni, G. Cerullo, Opt. Express 2016, 24, 28491

[26] G. Auböck, C. Consani, F. van Mourik, M. Chergui, Opt. Lett. 2012, 37, 2337.

[27] G. Auböck, C. Consani, R. Monni, A. Cannizzo, F. van Mourik, M. Chergui, Rev. Sci. Instrum 2012, 83, 093105

[28] P. Baum, S. Lochbrunner, E. Riedle, Opt. Lett. 2004, 29, 1686

[29] E. Baldini, T. Palmieri, E. Pomarico, G. Auböck, M. Chergui, arXiv:1703.07818, submitted to Nano Lett.

[30] A. Cannizzo, O. Bräm, G. Zgrablic, A Tortschanoff, A. Ajdarzadeh Oskouei, F van Mourik, M. Chergui, Opt. Lett. 2007, 32, 3555

31] C. Bonati, A Cannizzo, D Tonti, A. Tortschanoff, F. van Mourik, M. Chergui, Phys. Rev. B 2007, 76, 033304

[32] J. R. Lakowicz, 'Principles of Fluorescence Spectroscopy', Kluwer Academic/Plenum, New York, 1999.

[33] J. A. Stevens, J. J. Link, Y. T. Kao, C. Zang, L. J. Wang, D. P. Zhong, J. Phys. Chem. B 2010, 114, 1498.

[34] S. Schenkl, F. van Mourik, G. van der Zwan, S. Haacke, M. Chergui, Science 2005, 309, 917.

[35] J. Leonard, E. Portuondo-Campa, A. Cannizzo, F. van Mourik, G. van der Zwan, J. Tittor, S. Haacke, M. Chergui, Proc. Natl. Acad. Sci. U.S.A. 2009, 106, 7718

[36] O. Bräm, C. Consani, A. Cannizzo, M. Chergui, J. Phys. Chem. B 2011, 115, 13723.

[37] C. Consani, O. Bräm, F. van Mourik, A. Cannizzo, M. Chergui, Chem. Phys. 2012, 396, 108.

[38] C. Consani, G. Auböck, O. Bräm, F. van Mourik, M. Chergui, J. Chem. Phys. 2014, 140, 025103 .

[39] C. Consani, G. Auböck, F. van Mourik, M Chergui, Science 2013, 339, 1586

[40] R. Monni, A. Al Haddad, F. van Mourik, G. Auböck, M. Chergui, Proc. Natl. Acad. Sci. U.S.A 2015, 112, 5602 .
[41] R. Schweitzer-Stenner, J. Phys. Chem. B 2008, 112, 10358.

[42] D. Eden, J. B. Matthew, J. J. Rosa, F. M. Richards, Proc. Natl. Acad. Sci. U.S.A. 1982, $79,815$.

[43] G. Weber, F. J. W. Teale, Discuss. Faraday Soc. 1959, 27, 134.

[44] K. J. Willis, A. G. Szabo, M. Zuker, J. M. Ridgeway, B. Alpert, Biochemistry 1990, 29. 5270

[45] R. M. Hochstrasser, D. K. Negus, Proc. Natl. Acad. Sci. U.S.A. 1984, 81, 4399.

[46] C. J. Suess, J. D. Hirst, N. A. Besley, J. Comput. Chem. 2017, DOI: $10.1002 /$ jcc. 24793

[47] F. Messina, E. Pomarico, M. Silatani, E. Baranoff, M. Chergui, J. Phys. Chem. Lett. 2015, 6, 4475.

[48] A. Sarkar, S. Chakravorti, Chem. Phys. Lett. 1995, 235, 195 .

[49] O. Bräm, F. Messina, A. M. El-Zohry, A Cannizzo, M. Chergui, Chem. Phys. 2012, 393 , 51

[50] M. Chergui, Acc. Chem. Res. 2015, 48, 801

[51] E. Baldini, L. Chiodo, A. Dominguez, M Palummo, S. Moser, M. Yazdi, G. Auböck, B. P. P. Mallett, H. Berger, A. Magrez, C. Bernhard, M. Grioni, A. Rubio, M. Chergui, Nat. Commun. 2017, 8, 13 .

[52] P. Zu, Z. K. Tang, G. K. L. Wong, M. Kawasaki, A. Ohtomo, H. Koinuma, Y. Segawa, Solid State Commun. 1997, 103, 459.

[53] R. Newmann, R. M. Chrenko, Phys. Rev. 1959. 114, 1507.

[54] C. Taranto, G. Sangiovanni, K. Held, M. Capone, A. Georges, A. Toschi, Phys. Rev. B 2012, 85, 085124.

[55] A. E. Bragg, M. C. Cavanagh, B. J. Schwartz, Science 2008, 321, 1817.

[56] X. Chen, S. E. Bradforth, Annu. Rev. Phys. Chem. 2008, 59, 203.

[57] J. A. Kloepfer, V. H. Vilchiz, V. A. Lenchenkov, S. E. Bradforth, Chem. Phys. Lett. 1998, 298, 120.

[58] A. Lübcke, F. Buchner, N. Heine, I. V. Hertel, I. V. Hertel, T. Schultz, Phys. Chem. Chem. Phys. 2010, 12, 14629

[59] Y. Tang, H. Shen, K. Sekiguchi, N. Kurahashi, T. Mizuno, Y.-I. Suzuki, T. Suzuki, Phys. Chem. Chem. Phys. 2010, 12, 3653.

[60] F. Messina, O. Bräm, A. Cannizzo, M. Chergui, Nature Comm. 2013, 4, 2119.

[61] M. Reinhard, G. Auböck, N. A. Besley, I. P. Clark, M. W. D. Hanson-Heine, R. Horvath, T. S. Murphy, T. J. Penfold, M. Towrie, M. W. George, M. Chergui, J. Am. Chem. Soc. 2017, under review.

[62] A. Picchiotti, V. I. Prokhorenko, R. J. D. Miller, Rev. Sci. Instrum. 2015, 86, 093105 